\title{
Singularity problems to fourth-order Rayleigh equation with time-dependent deviating argument
}

Yun Xin' and Hongmin Liư ${ }^{1 *}$

"Correspondence:

hongminliu_1982@126.com

${ }^{1}$ College of Computer Science and

Technology, Henan Polytechnic

University, Jiaozuo, China

\begin{abstract}
The paper is devoted to an investigation of the existence of a positive periodic solution for a kind of fourth-order singular Rayleigh equation with time-dependent deviating argument, where the nonlinear term $g$ satisfies singularities of attractive and repulsive type at the origin and has time-dependent deviating argument. By applications of coincidence degree theory, we prove that this equation has at least one positive periodic solution. At last, two examples are given to show applications of theorems.
\end{abstract}

MSC: 34B16; 34C25

Keywords: Positive periodic solution; Fourth-order Rayleigh equation; Singularities of attractive and repulsive type; Time-dependent deviating argument

\section{Introduction}

In this paper, we consider the following fourth-order $p$-Laplacian singular Rayleigh equation with time-dependent deviating argument:

$$
\left(\phi_{p}\left(x^{\prime \prime}(t)\right)\right)^{\prime \prime}+f\left(t, x^{\prime}(t)\right)+g(t, x(t-\delta(t)))=e(t)
$$

where $\phi_{p}: \mathbb{R} \rightarrow \mathbb{R}$ is given by $\phi_{p}(s)=|s|^{p-2} s$, and $p>1$ is a constant; $f: \mathbb{R} \times \mathbb{R} \rightarrow \mathbb{R}$ is a continuous $T$-periodic function about $t$ and $f(t, 0)=0 ; e: \mathbb{R} \rightarrow \mathbb{R}$ is a continuous periodic functions with $e(t+T) \equiv e(t) ; \delta \in C^{1}(\mathbb{R}, \mathbb{R})$ is a $T$-periodic function; $g: \mathbb{R} \times(0,+\infty) \rightarrow \mathbb{R}$ is a $L^{2}$-Carathéodory function defined on $\mathbb{R}^{2}$, and $g(t, \cdot)=g(t+T, \cdot)$; it is said that Eq. (1.1) is singularity of attractive type (resp. repulsive type) if $g(t, x) \rightarrow+\infty$ (resp. $g(t, x) \rightarrow-\infty$ ) as $x \rightarrow 0^{+}$for $t \in \mathbb{R}$.

As is well known, the Rayleigh equation can be derived from many fields, such as engineering technique, physics and mechanics fields, and an important question is whether we have periodic solutions to the Rayleigh equation. Gaines and Mawhin [1] in 1977 introduced continuation theorems and applied this theorem to prove the existence of a periodic solutions for the Rayleigh equation ([1], p. 99)

$$
x^{\prime \prime}(t)+f\left(x^{\prime}(t)\right)+g(t, x(t))=0 .
$$

(c) The Author(s) 2018. This article is distributed under the terms of the Creative Commons Attribution 4.0 International License (http://creativecommons.org/licenses/by/4.0/), which permits unrestricted use, distribution, and reproduction in any medium, provided you give appropriate credit to the original author(s) and the source, provide a link to the Creative Commons license, and indicate if changes were made. 
Gaines and Mawhin's work has attracted the attention of many scholars to the Rayleigh equation. More recently, the method of lower and upper solutions [2], Mawhin's continuous theorem [3-5], topological degree and time maps [6, 7], and the Manásevich-Mawhin continuation theorem [8-10] have been employed to investigate the existence of a periodic solution of Rayleigh equations.

On the other hand, the existence of a periodic solutions for a singular differential equation was extensively studied (see [11-20]). Among these, there are some results on the Liénard equation with a singularity of repulsive type [14, 15, 19, 20]. Zhang [19] in 1996 introduced the problem of periodic solutions of the following Liénard equation with a repulsive singularity:

$$
x^{\prime \prime}(t)+f(x(t)) x^{\prime}(t)+g(t, x(t))=0
$$

where the nonlinear term $g$ has a singularity of repulsive type at the origin and satisfies semilinear condition at $x=\infty$, the author proved that Eq. (1.3) has at least one positive $T$-periodic solution by using Mawhin's continuous theorem. Wang [20] in 2014 improved Eq. (1.3) and proved the existence of a positive periodic solution of the following Liénard equation with a singularity of repulsive type and a deviating argument:

$$
x^{\prime \prime}(t)+f(x(t)) x^{\prime}(t)+g(t, x(t-\delta))=0,
$$

where $\delta$ is a constant and $\delta \in[0, T]$.

Nowadays, a good deal of work has been performed on the existence of a positive periodic solution of the Rayleigh equation with a singularity [21-24]. Wang and Ma [24] in 2015 discussed a kind of singular Rayleigh equation as follows:

$$
x^{\prime \prime}(t)+f\left(t, x^{\prime}(t)\right)+g(x(t))=p(t)
$$

where the nonlinear term $g$ has a singularity of repulsive type at the origin and satisfies semilinear condition. The authors obtained the existence of a positive periodic solution for Eq. (1.5) by applications of the limit properties of time map. Lu and Chen [21] in 2017 studied the following Rayleigh equation with singularity of repulsive type:

$$
x^{\prime \prime}+f\left(t, x^{\prime}(t)\right)+\varphi(t) x(t)-\frac{1}{x^{\gamma}(t)}=p(t)
$$

where $\gamma \geq 1$. By using topological degree theory, the authors proved Eq. (1.6) has at least one positive $T$-periodic solution.

Inspired by the above paper [1, 19-21, 24], in this paper, we further consider the existence of a positive $T$-periodic solution for Eq. (1.1) with singularities of attractive and repulsive type. By applications of coincidence degree theory, we obtain the following conclusions.

Theorem 1.1 Assume that the following conditions hold:

$\left(H_{1}\right)$ There exists a positive constant $N$ such that

$$
|f(t, u)| \leq N, \quad \text { for }(t, u) \in[0, T] \times \mathbb{R} .
$$


$\left(H_{2}\right)$ There exist two positive constants $D_{1}, D_{2}$ with $D_{1}<D_{2}$ such that $g(t, x)-e(t)<-N$ for all $(t, x) \in[0, T] \times\left(0, D_{1}\right)$, and $g(t, x)-e(t)>N$ for all $(t, x) \in[0, T] \times\left(D_{2},+\infty\right)$.

$\left(H_{3}\right)$ There exist positive constants $a, b$ such that

$$
g(t, x) \leq a x^{p-1}+b, \quad \text { for all }(t, x) \in[0, T] \times(0,+\infty) .
$$

$\left(H_{4}\right) g(t, x)=g_{0}(x)+g_{1}(t, x)$, where $g_{0} \in C((0, \infty) ; \mathbb{R})$ and $g_{1}:[0, T] \times[0, \infty) \rightarrow \mathbb{R}$ is an $L^{2}$-Carathéodory function.

$\left(H_{5}\right)$ (Singularity of repulsive type)

$$
\int_{0}^{1} g_{0}(x) d x=-\infty
$$

Then Eq. (1.1) has at least one positive T-periodic solution if

$$
\begin{aligned}
& 0<\frac{a T}{1-\delta^{\prime}}\left(\frac{T}{\pi_{p}}\right)^{2 p-1}<1, \\
& \text { where } \delta^{\prime}=: \max _{t \in[0, T]}|\delta(t)| \text {, and } 1 \leq p<\infty, \pi_{p}=2 \int_{0}^{(p-1) / p} \frac{d s}{\left(1-\frac{s^{p}}{p-1}\right)^{1 / p}}=\frac{2 \pi(p-1)^{1 / p}}{p \sin (\pi / p)}
\end{aligned}
$$

Remark 1.2 The friction term $f(x(t)) x^{\prime}(t)$ in Eqs. (1.3) and (1.4) satisfy $\int_{0}^{T} f(x(t)) x^{\prime}(t) d t=0$, which is crucial to estimate $a$ priori bounds of a positive $T$-periodic solution for these equations. However, in this paper, the friction term $f\left(t, x^{\prime}\right)$ may not satisfy $\int_{0}^{T} f\left(t, x^{\prime}(t)\right) d t=$ 0 . For example, let

$$
f\left(t, x^{\prime}\right)=\left(\cos ^{2}(2 t)+100\right) \sin x^{\prime}(t)
$$

Obviously, $\int_{0}^{T}\left(\cos ^{2}(2 t)+100\right) \sin x^{\prime}(t) \neq 0$. This implies that our methods to estimate $a$ priori bounds of a positive $T$-periodic solution for Eq. (1.1) is more complex than Eqs. (1.3) and (1.4).

Remark 1.3 From Eqs. (1.3), (1.4), (1.5), (1.6) in [19-21, 24], the nonlinear term $g$ has a deviating argument (i.e., $\delta$ is a positive constant and $0 \leq \delta<T$ ). But, in this paper, the nonlinear term $g$ satisfies a time-dependent deviating argument. For example, let

$$
\delta(t)=\frac{1}{4} \sin 2 t
$$

Obviously, the work on estimating a lower bounds of a positive $T$-periodic solution for Eq. (1.1) is more difficult than the corresponding work on Eqs. (1.3), (1.4), (1.5), (1.6). Therefore, we have to find another way to get over the difficulty.

Remark 1.4 If Eq. (1.1) satisfies singularity of attractive type, i.e., $\int_{0}^{1} g_{0}(x) d x=+\infty$. Obviously, attractive condition and $\left(H_{2}\right),\left(H_{3}\right),\left(H_{5}\right)$ are contradiction. Therefore, the above method and conditions are no long applicable to the proof of existence of a positive periodic solution for Eq. (1.1) with singularity of attractive type. Next, we give other conditions to prove the existence of a positive $T$-periodic solution for Eq. (1.1) with singularity of attractive type. 
Theorem 1.5 Assume that conditions $\left(H_{1}\right)$ and $\left(H_{4}\right)$ hold. Suppose the following conditions are satisfied:

$\left(H_{6}\right)$ There exist two positive constants $D_{3}, D_{4}$ with $D_{3}<D_{4}$ such that $g(t, x)-e(t)>N$ for all $(t, x) \in[0, T] \times\left(0, D_{3}\right)$, and $g(t, x)-e(t)<-N$ for all $(t, x) \in[0, T] \times\left(D_{4},+\infty\right)$.

$\left(H_{7}\right)$ There exist positive constants $a^{\prime}, b^{\prime}$ such that

$$
-g(t, x) \leq a^{\prime} x^{p-1}+b^{\prime}, \quad \text { for all }(t, x) \in[0, T] \times(0,+\infty)
$$

$\left(H_{8}\right)$ (Singularity of attractive type)

$$
\int_{0}^{1} g_{0}(x) d x=+\infty
$$

Then Eq. (1.1) has at least one positive T-periodic solution if

$$
0<\frac{a^{\prime} T}{1-\delta^{\prime}}\left(\frac{T}{\pi_{p}}\right)^{2 p-1}<1
$$

\section{Preparation}

Lemma 2.1 (Gaines and Mawhin [1]) Suppose that $X$ and $Y$ are two Banach spaces, and $L: D(L) \subset X \rightarrow Y$ is a Fredholm operator with index zero. Let $\Omega \subset X$ be an open bounded set and $N: \bar{\Omega} \rightarrow Y$ be L-compact on $\bar{\Omega}$. Assume that the following conditions hold:

(1) $L x \neq \lambda N x, \forall x \in \partial \Omega \cap D(L), \lambda \in(0,1)$;

(2) $N x \notin \operatorname{Im} L, \forall x \in \partial \Omega \cap \operatorname{Ker} L$;

(3) $\operatorname{deg}\{J Q N, \Omega \cap \operatorname{Ker} L, 0\} \neq 0$, where $J: \operatorname{Im} Q \rightarrow \operatorname{Ker} L$ is an isomorphism.

Then the equation $L x=N x$ has a solution in $\bar{\Omega} \cap D(L)$.

Lemma $2.2([25])$ If $\omega \in C^{1}(\mathbb{R}, \mathbb{R})$ and $\omega(0)=\omega(T)=0$, then

$$
\left(\int_{0}^{T}|\omega(t)|^{p} d t\right)^{\frac{1}{p}} \leq\left(\frac{T}{\pi_{p}}\right)\left(\int_{0}^{T}\left|\omega^{\prime}(t)\right|^{p} d t\right)^{\frac{1}{p}}
$$

In order to apply topological degree theorem to study the existence of a positive periodic solution for Eq. (1.1), we rewrite Eq. (1.1) in the form:

$$
\left\{\begin{array}{l}
x_{1}^{\prime \prime}(t)=\left(\phi_{q}\left(x_{2}(t)\right)\right. \\
x_{2}^{\prime \prime}(t)=-f\left(t, x_{1}^{\prime}(t)\right)-g\left(t, x_{1}(t-\delta(t))\right)+e(t),
\end{array}\right.
$$

where $\frac{1}{p}+\frac{1}{q}=1$. Clearly, if $x(t)=\left(x_{1}(t), x_{2}(t)\right)^{\top}$ is a $T$-periodic solution to Eq. (2.1), then $x_{1}(t)$ must be a $T$-periodic solution to Eq. (1.1). Thus, the problem of finding a $T$-periodic solution for Eq. (1.1) reduces to finding one for Eq. (2.1).

Let

$$
X:=\left\{x=\left(x_{1}(t), x_{2}(t)\right) \in C^{2}\left(\mathbb{R}, \mathbb{R}^{2}\right): x(t+T)-x(t) \equiv 0\right\}
$$

with the norm $\|x\|:=\max \left\{\left\|x_{1}\right\|,\left\|x_{2}\right\|\right\}$;

$$
Y:=\left\{x=\left(x_{1}(t), x_{2}(t)\right) \in C^{1}\left(\mathbb{R}, \mathbb{R}^{2}\right): x(t+T)-x(t) \equiv 0\right\}
$$


with the norm $\|x\|_{\infty}:=\max \left\{\|x\|,\left\|x^{\prime}\right\|\right\}$. Clearly, $X$ and $Y$ are both Banach spaces. Meanwhile, define

$$
L: D(L) \subset X \rightarrow Y, \quad \text { by }(L x)(t)=\left(\begin{array}{l}
x_{1}^{\prime \prime}(t) \\
x_{2}^{\prime \prime}(t)
\end{array}\right),
$$

where $D(L)=\left\{x=\left(x_{1}, x_{2}\right)^{\top} \in C^{2}\left(\mathbb{R}, \mathbb{R}^{2}\right): x(t+T)-x(t) \equiv 0, t \in \mathbb{R}\right\}$. Define a nonlinear operator $N: X \rightarrow Y$ as follows:

$$
(N x)(t)=\left(\begin{array}{c}
\phi_{q}\left(x_{2}(t)\right) \\
-f\left(t, x_{1}^{\prime}(t)\right)-g\left(t, x_{1}(t-\delta(t))\right)+e(t)
\end{array}\right) .
$$

Then Eq. (2.1) can be converted to the abstract equation $L x=N x$.

From the definition of $L$, one can easily see that

$$
\operatorname{Ker} L \cong \mathbb{R}^{2}, \quad \operatorname{Im} L=\left\{y \in Y: \int_{0}^{T}\left(\begin{array}{l}
y_{1}(s) \\
y_{2}(s)
\end{array}\right) d s=\left(\begin{array}{l}
0 \\
0
\end{array}\right)\right\}
$$

So $L$ is a Fredholm operator with index zero. Let $P: X \rightarrow \operatorname{Ker} L$ and $Q: Y \rightarrow \operatorname{Im} Q \subset \mathbb{R}^{2}$ be defined by

$$
P x:=\left(\begin{array}{c}
\left(x_{1}\right)(0) \\
x_{2}(0)
\end{array}\right) ; \quad Q y:=\frac{1}{T} \int_{0}^{T}\left(\begin{array}{l}
y_{1}(s) \\
y_{2}(s)
\end{array}\right) d s,
$$

then $\operatorname{Im} P=\operatorname{Ker} L, \operatorname{Ker} Q=\operatorname{Im} L$. let $K$ denote the inverse of $\left.L\right|_{\operatorname{Ker} p \cap D(L)}$. It is easy to see that $\operatorname{Ker} L=\operatorname{Im} Q=\mathbb{R}^{2}$ and

$$
[K y](t)=\operatorname{col}\left(\int_{0}^{T} G_{1}(t, s) y_{1}(s) d s, \int_{0}^{T} G_{2}(t, s) y_{2}(s) d s\right)
$$

where

$$
G_{i}(t, s)= \begin{cases}\frac{-s(T-t)}{T}, & 0 \leq s \leq t \leq T, \quad i=1,2 . \\ \frac{-t(T-s)}{T}, & 0 \leq t<s \leq T,\end{cases}
$$

\section{Main results}

In the section, we first consider the existence of a positive $T$-periodic solution for Eq. (1.1) with singularity of repulsive type.

Proof of Theorem 1.1 Consider the operator equation

$$
L x=\lambda N x, \quad \lambda \in(0,1)
$$

where $L$ and $N$ are defined by Eqs. (2.2) and (2.4). Set

$$
\Omega_{1}=\{x: L x=\lambda N x, \lambda \in(0,1)\} .
$$


If $x(t)=\left(x_{1}(t), x_{2}(t)\right)^{\top} \in \Omega_{1}$, then

$$
\left\{\begin{array}{l}
x_{1}^{\prime \prime}(t)=\lambda \phi_{q}\left(x_{2}(t)\right) \\
x_{2}^{\prime \prime}(t)=-\lambda f\left(t, x_{1}^{\prime}(t)\right)-\lambda g\left(t, x_{1}(t-\delta(t))\right)+\lambda e(t) .
\end{array}\right.
$$

Substituting $x_{2}(t)=\frac{1}{\lambda^{p-1}}\left(\phi_{p}\left(x_{1}\right)^{\prime \prime}(t)\right)$ into the second equation of (3.1)

$$
\phi_{p}\left(x_{1}^{\prime \prime}(t)\right)^{\prime \prime}(t)+\lambda^{p} f\left(t, x_{1}^{\prime}(t)\right)+\lambda^{p} g\left(t, x_{1}(t-\delta(t))\right)=\lambda^{p} e(t) .
$$

Integrating both side of Eq. (3.2) over [0,T], we have

$$
\int_{0}^{T}\left(f\left(t, x_{1}^{\prime}(t)\right)+g\left(t, x_{1}(t-\delta(t))\right)-e(t)\right) d t=0
$$

since $\int_{0}^{T}\left(\phi_{p}\left(A x_{1}\right)^{\prime \prime}(t)\right)^{\prime \prime}=0$. From Eq. (3.3) and condition $\left(H_{1}\right)$, we deduce

$$
-N T \leq \int_{0}^{T}\left(g\left(t, x_{1}(t-\delta(t))\right)-e(t)\right) d t \leq N T .
$$

Then, by condition $\left(H_{2}\right)$, we know that there are two points $\xi, \eta \in(0, T)$ such that

$$
x_{1}(\xi-\delta(\xi)) \geq D_{1}, \quad x_{1}(\eta) \leq D_{2} .
$$

Then, from Eq. (3.4), we have

$$
\begin{aligned}
x(t) & =\frac{1}{2}(x(t)+x(t-T)) \\
& =\frac{1}{2}\left(x(\eta)+\int_{\eta}^{t} x^{\prime}(s) d s+x(\eta)-\int_{t-T}^{\eta} x^{\prime}(s) d s\right) \\
& =x(\eta)+\frac{1}{2} \int_{t-T}^{t} x^{\prime}(s) d s \\
& \leq D_{2}+\frac{1}{2} \int_{0}^{T}\left|x^{\prime}(t)\right| d t .
\end{aligned}
$$

Multiplying both sides of Eq. (3.2) by $x_{1}(t)$ and integrating over the interval $[0, T]$, we get

$$
\begin{aligned}
& \int_{0}^{T} \phi_{p}\left(x_{1}^{\prime \prime}(t)\right)^{\prime \prime} x_{1}(t) d t+\lambda^{p} \int_{0}^{T} f\left(t, x_{1}^{\prime}(t)\right) x_{1}(t) d t+\lambda^{p} \int_{0}^{T} g\left(t, x_{1}(t-\delta(t))\right) x_{1}(t) d t \\
& \quad=\lambda^{p} \int_{0}^{T} e(t) x_{1}(t) d t .
\end{aligned}
$$

Substituting $\int_{0}^{T} \phi_{p}\left(x_{1}^{\prime \prime}(t)\right)^{\prime \prime} x_{1}(t) d t=\int_{0}^{T}\left|x_{1}^{\prime \prime}(t)\right|^{p} d t$ into Eq. (3.6), we arrive at

$$
\begin{aligned}
\int_{0}^{T}\left|x_{1}^{\prime \prime}(t)\right|^{p} d t= & -\lambda^{p} \int_{0}^{T} f\left(t, x_{1}^{\prime}(t)\right) x_{1}(t) d t-\lambda^{p} \int_{0}^{T} g\left(t, x_{1}(t-\delta(t))\right) x_{1}(t) d t \\
& +\lambda^{p} \int_{0}^{T} e(t) x_{1}(t) d t .
\end{aligned}
$$


Therefore, from condition $\left(H_{1}\right)$, we deduce

$$
\begin{aligned}
\int_{0}^{T}\left|x_{1}^{\prime \prime}(t)\right|^{p} d t \leq & \int_{0}^{T}\left|f\left(t, x_{1}^{\prime}(t)\right)\right|\left|x_{1}(t)\right| d t+\int_{0}^{T}\left|g\left(t, x_{1}(t-\delta(t))\right)\right|\left|x_{1}(t)\right| d t \\
& +\int_{0}^{T}|e(t)|\left|x_{1}(t)\right| d t \\
\leq & \left\|x_{1}\right\| N T+\left\|x_{1}\right\| \int_{0}^{T}\left|g\left(t, x_{1}(t-\delta(t))\right)\right| d t+\|e\| T\left\|x_{1}\right\|,
\end{aligned}
$$

where $\|e\|:=\max _{t \in[0, T]}|e(t)|$. From conditions $\left(H_{1}\right),\left(H_{2}\right)$ and Eq. (3.3), we obtain

$$
\begin{aligned}
& \int_{0}^{T}\left|g\left(t, x_{1}(t-\delta(t))\right)\right| d t \\
& \quad=\int_{g\left(t, x_{1}\right) \geq 0} g\left(t, x_{1}(t-\delta(t))\right) d t-\int_{g\left(t, x_{1}\right) \leq 0} g\left(t, x_{1}(t-\delta(t))\right) d t \\
& \quad=2 \int_{g\left(t, x_{1}\right) \geq 0} g\left(t, x_{1}(t-\delta(t))\right) d t+\int_{0}^{T} f\left(t, x_{1}^{\prime}(t)\right) d t-\int_{0}^{T} e(t) d t \\
& \quad \leq 2 a \int_{0}^{T} x_{1}^{p-1}(t-\delta(t)) d t+2 b T+\int_{0}^{T}\left|f\left(t, x_{1}^{\prime}(t)\right)\right| d t+\int_{0}^{T}|e(t)| d t \\
& \quad=2 a \int_{0}^{T}\left|x_{1}(t-\delta(t))\right|^{p-1} d t+2 b T+N T+\|e\| T \\
& \quad \leq \frac{2 a}{1-\delta^{\prime}} \int_{0}^{T}\left|x_{1}(t)\right|^{p-1} d t+2 b T+N T+\|e\| T,
\end{aligned}
$$

from $\frac{a T}{1-\delta^{\prime}}\left(\frac{T}{\pi_{p}}\right)^{2 p-1}>0$, we know that $\delta^{\prime}<1$. Substituting Eqs. (3.5) and (3.8) into (3.7), and by using the Hölder inequality, we see that

$$
\begin{aligned}
\int_{0}^{T}\left|x_{1}^{\prime \prime}(t)\right|^{p} d t \leq & \frac{2 a}{1-\delta^{\prime}}\left\|x_{1}\right\| \int_{0}^{T}\left|x_{1}(t)\right|^{p} d t+2 T(N+b+\|e\|)\left\|x_{1}\right\| \\
\leq & \frac{2 a}{1-\delta^{\prime}}\left(D_{2}+\frac{1}{2} \int_{0}^{T}\left|x_{1}^{\prime}(t)\right| d t\right) \int_{0}^{T}\left|x_{1}(t)\right|^{p-1} d t \\
& +F_{1}\left(D_{2}+\frac{1}{2} \int_{0}^{T}\left|x_{1}^{\prime}(t)\right| d t\right) \\
\leq & \frac{a T}{1-\delta^{\prime}}\left(\int_{0}^{T}\left|x_{1}^{\prime}(t)\right|^{p} d t\right)^{\frac{1}{p}}\left(\int_{0}^{T}\left|x_{1}(t)\right|^{p} d t\right)^{\frac{p-1}{p}} \\
& +\frac{2 a D_{2} T^{\frac{1}{p}}}{1-\delta^{\prime}}\left(\int_{0}^{T}\left|x_{1}(t)\right|^{p} d t\right)^{\frac{p-1}{p}} \\
& +\frac{F_{1} T^{\frac{1}{q}}}{2}\left(\int_{0}^{T}\left|x_{1}^{\prime}(t)\right|^{p} d t\right)^{\frac{1}{p}}+F_{1} D_{2},
\end{aligned}
$$

where $F_{1}:=2(N+b+\|e\|) T$. 
Let $\omega(t)=x_{1}(t+\eta)-x_{1}(\eta)$, here $x_{1}(\eta) \leq D_{2}$, and then $\omega(0)=\omega(T)=0$. By Lemma 2.2 and the Minkowski inequality [26], it is clear that

$$
\begin{aligned}
\left(\int_{0}^{T}\left|x_{1}(t)\right|^{p} d t\right)^{\frac{1}{p}} & =\left(\int_{0}^{T}\left|\omega(t)+x_{1}(\xi)\right|^{p} d t\right)^{\frac{1}{p}} \\
& \leq\left(\int_{0}^{T}|\omega(t)|^{p} d t\right)^{\frac{1}{p}}+\left(\int_{0}^{T}\left|x_{1}(\eta)\right|^{p} d t\right)^{\frac{1}{p}} \\
& \leq\left(\frac{T}{\pi_{p}}\right)\left(\int_{0}^{T}\left|\omega^{\prime}(t)\right|^{p} d t\right)^{\frac{1}{p}}+D_{2} T^{\frac{1}{p}} \\
& =\left(\frac{T}{\pi_{p}}\right)\left(\int_{0}^{T}\left|x_{1}^{\prime}(t)\right|^{p} d t\right)^{\frac{1}{p}}+D_{2} T^{\frac{1}{p}}
\end{aligned}
$$

On the other hand, in view of $x_{1}(0)=x_{1}(T)$, there exists a point $t_{1} \in(0, T)$ such that $x_{1}^{\prime}\left(t_{1}\right)=0$. Let $\omega_{*}(t)=x_{1}^{\prime}\left(t+t_{1}\right)$, it is easy to see that $\omega_{*}(0)=\omega_{*}(T)=0$ for $\omega_{*} \in C^{1}(\mathbb{R}, \mathbb{R})$. By Lemma 2.2,

$$
\begin{aligned}
\left(\int_{0}^{T}\left|x_{1}^{\prime}(t)\right|^{p} d t\right)^{\frac{1}{p}} & =\left(\int_{0}^{T}\left|\omega_{*}(t)\right|^{p} d t\right)^{\frac{1}{p}} \leq\left(\frac{T}{\pi_{p}}\right)\left(\int_{0}^{T}\left|\omega_{*}^{\prime}(t)\right|^{p} d t\right)^{\frac{1}{p}} \\
& =\left(\frac{T}{\pi_{p}}\right)\left(\int_{0}^{T}\left|x_{1}^{\prime \prime}(t)\right|^{p} d t\right)^{\frac{1}{p}} .
\end{aligned}
$$

Substituting Eqs. (3.11) into (3.10), we obtain

$$
\left(\int_{0}^{T}\left|x_{1}(t)\right|^{p} d t\right)^{\frac{1}{p}} \leq\left(\frac{T}{\pi_{p}}\right)^{2}\left(\int_{0}^{T}\left|x_{1}^{\prime \prime}(t)\right|^{p} d t\right)^{\frac{1}{p}}+D_{2} T^{\frac{1}{p}} .
$$

Furthermore, substituting Eqs. (3.11) and (3.12) into (3.9), we see that

$$
\begin{aligned}
\int_{0}^{T}\left|\left(x_{1}\right)^{\prime \prime}(t)\right|^{p} d t & \\
\leq & \frac{a T}{1-\delta^{\prime}}\left(\frac{T}{\pi_{p}}\right)\left(\int_{0}^{T}\left|x_{1}^{\prime \prime}(t)\right|^{p} d t\right)^{\frac{1}{p}}\left(\left(\frac{T}{\pi_{p}}\right)^{2}\left(\int_{0}^{T}\left|x_{1}^{\prime \prime}(t)\right|^{p} d t\right)^{\frac{1}{p}}+D_{2} T^{\frac{1}{p}}\right)^{p-1} \\
& +\frac{2 a D_{2} T^{\frac{1}{p}}}{1-\delta^{\prime}}\left(\left(\frac{T}{\pi_{p}}\right)^{2}\left(\int_{0}^{T}\left|x_{1}^{\prime \prime}(t)\right|^{p} d t\right)^{\frac{1}{p}}+D_{2} T^{\frac{1}{p}}\right)^{p-1} \\
& +\frac{F_{1} T^{\frac{1}{q}}}{2}\left(\frac{T}{\pi_{p}}\right)\left(\int_{0}^{T}\left|x_{1}^{\prime \prime}(t)\right|^{p} d t\right)^{\frac{1}{p}}+F_{1} D_{2} \\
= & \frac{a T}{1-\delta^{\prime}}\left(\frac{T}{\pi_{p}}\right)^{2 p-1}\left(1+\frac{D_{2} T^{\frac{1}{p}}}{\left(\frac{T}{\pi_{p}}\right)^{2}\left(\int_{0}^{T}\left|x_{1}^{\prime \prime}(t)\right|^{p} d t\right)^{\frac{1}{p}}}\right)^{p-1} \int_{0}^{T}\left|x_{1}^{\prime \prime}(t)\right|^{p} d t \\
& +\frac{2 a D_{2} T^{\frac{1}{p}}}{1-\delta^{\prime}}\left(\frac{T}{\pi_{p}}\right)^{2 p-2}\left(1+\frac{D_{2} T^{\frac{1}{p}}}{\left(\frac{T}{\pi_{p}}\right)^{2}\left(\int_{0}^{T}\left|x_{1}^{\prime \prime}(t)\right|^{p} d t\right)^{\frac{1}{p}}}\right)^{p-1}\left(\int_{0}^{T}\left|x_{1}^{\prime \prime}(t)\right|^{p} d t\right)^{\frac{p-1}{p}} \\
& +\frac{F_{1} T^{\frac{1}{q}}}{2}\left(\frac{T}{\pi_{p}}\right)\left(\int_{0}^{T}\left|x_{1}^{\prime \prime}(t)\right|^{p} d t\right)^{\frac{1}{p}}+F_{1} D_{2} .
\end{aligned}
$$


Next, we introduce a classical inequality, there exists a $k(p)>0$ which is dependent on $p$ only,

$$
(1+x)^{p} \leq 1+(1+p) x, \quad \text { for } x \in[0, k(p)] .
$$

Then, we consider the following two cases:

Case 1. If $\frac{D_{2} T^{\frac{1}{p}}}{\left(\frac{T}{\pi_{p}}\right)^{2}\left(\int_{0}^{T}\left|x_{1}^{\prime \prime}(t)\right|^{p} d t\right)^{\frac{1}{p}}}>k(p)$, then it is obvious that

$$
\left(\int_{0}^{T}\left|x_{1}^{\prime \prime}(t)\right|^{p} d t\right)^{\frac{1}{p}}<\frac{D_{2} T^{\frac{1}{p}}}{k(p)}\left(\frac{T}{\pi_{p}}\right)^{-2} .
$$

From Eqs. (3.5) and (3.11), by using the Hölder inequality, we deduce

$$
\begin{aligned}
x(t) & \leq D_{2}+\frac{1}{2} T^{\frac{1}{q}}\left(\int_{0}^{T}\left|x^{\prime}(t)\right|^{p} d t\right)^{\frac{1}{p}} \\
& \leq D_{2}+\frac{1}{2} T^{\frac{1}{q}}\left(\frac{T}{\pi_{p}}\right)\left(\int_{0}^{T}\left|x^{\prime \prime}(t)\right|^{p} d t\right)^{\frac{1}{p}} \\
& \leq D_{2}+\frac{T D_{2}}{2 k(p)}\left(\frac{T}{\pi_{p}}\right)^{-1}:=M_{1} .
\end{aligned}
$$

Case 2. If $\frac{D_{2} T^{\frac{1}{p}}}{\left(\frac{T}{\pi_{p}}\right)^{2}\left(\int_{0}^{T}\left|x_{1}^{\prime \prime}(t)\right|^{p} d t\right)^{\frac{1}{p}}}<k(p)$, from Eqs. (3.13) and (3.14), we obtain

$$
\begin{aligned}
\int_{0}^{T}\left|\left(x_{1}\right)^{\prime \prime}(t)\right|^{p} d t & \\
\leq & \frac{a T}{1-\delta^{\prime}}\left(\frac{T}{\pi_{p}}\right)^{2 p-1}\left(1+\frac{D_{2} T^{\frac{1}{p}} p}{\left(\frac{T}{\pi_{p}}\right)^{2}\left(\int_{0}^{T}\left|x_{1}^{\prime \prime}(t)\right|^{p} d t\right)^{\frac{1}{p}}}\right) \int_{0}^{T}\left|x_{1}^{\prime \prime}(t)\right|^{p} d t \\
& +\frac{2 a D_{2} T^{\frac{1}{p}}}{1-\delta^{\prime}}\left(\frac{T}{\pi_{p}}\right)^{2 p-2}\left(1+\frac{D_{2} T^{\frac{1}{p}} p}{\left(\frac{T}{\pi_{p}}\right)^{2}\left(\int_{0}^{T}\left|x_{1}^{\prime \prime}(t)\right|^{p} d t\right)^{\frac{1}{p}}}\right)\left(\int_{0}^{T}\left|x_{1}^{\prime \prime}(t)\right|^{p} d t\right)^{\frac{p-1}{p}} \\
& +\frac{F_{1} T^{\frac{1}{q}}}{2}\left(\frac{T}{\pi_{p}}\right)\left(\int_{0}^{T}\left|x_{1}^{\prime \prime}(t)\right|^{p} d t\right)^{\frac{1}{p}}+F_{1} D_{2} \\
= & \frac{a T}{1-\delta^{\prime}}\left(\frac{T}{\pi_{p}}\right)^{2 p-1} \int_{0}^{T}\left|x_{1}^{\prime \prime}(t)\right|^{p} d t+\frac{a D_{2} T^{1+\frac{1}{p}}}{1-\delta^{\prime}}\left(T p+2\left(\frac{T}{\pi}\right)\right)\left(\frac{T}{\pi_{p}}\right)^{2 p-2} \\
& \cdot\left(\int_{0}^{T}\left|x_{1}^{\prime \prime}(t)\right|^{p} d t\right)^{\frac{p-1}{p}}+\frac{2 a D_{2}^{2} T^{\frac{2}{p}} p}{1-\delta^{\prime}}\left(\frac{T}{\pi_{p}}\right)^{2 p-4}\left(\int_{0}^{T}\left|x_{1}^{\prime \prime}(t)\right|^{p} d t\right)^{\frac{p-2}{p}} \\
& +\frac{F_{1} T^{\frac{1}{q}}}{2}\left(\frac{T}{\pi_{p}}\right)\left(\int_{0}^{T}\left|x_{1}^{\prime \prime}(t)\right|^{p} d t\right)^{\frac{1}{p}}+F_{1} D_{2} .
\end{aligned}
$$

Since

$$
\frac{a T}{1-\delta^{\prime}}\left(\frac{T}{\pi_{p}}\right)^{2 p-1}<1,
$$


it is easy to see that there exists a positive constant $M_{1}^{\prime}$ such that

$$
\int_{0}^{T}\left|x_{1}^{\prime \prime}(t)\right|^{p} d t \leq M_{1}^{\prime}
$$

From Eq. (3.5) and Lemma 2.2, we have

$$
\begin{aligned}
x_{1}(t) & \leq D_{2}+\frac{1}{2} \int_{0}^{T}\left|x_{1}^{\prime}(t)\right| d t \\
& \leq D_{2}+\frac{1}{2} T^{\frac{1}{q}}\left(\frac{T}{\pi_{p}}\right)\left(\int_{0}^{T}\left|x_{1}^{\prime \prime}(t)\right|^{p} d t\right)^{\frac{1}{p}} \\
& \leq D_{2}+\frac{1}{2} T^{\frac{1}{q}}\left(\frac{T}{\pi_{p}}\right)\left(M_{1}^{\prime}\right)^{\frac{1}{p}}:=M_{1} .
\end{aligned}
$$

From Eq. (3.5), we have

$$
\begin{aligned}
\left\|x_{1}^{\prime}\right\| & \leq x_{1}^{\prime}\left(t_{1}\right)+\frac{1}{2} \int_{0}^{T}\left|x_{1}^{\prime \prime}(t)\right| d t \\
& \leq \frac{1}{2} T^{\frac{1}{q}}\left(\int_{0}^{T}\left|x_{1}^{\prime \prime}(t)\right| d t\right)^{\frac{1}{p}} \\
& \leq \frac{1}{2} T^{\frac{1}{q}} M_{1}^{\prime \frac{1}{p}}:=M_{2},
\end{aligned}
$$

since $x_{1}^{\prime}\left(t_{1}\right)=0$. From $x_{2}(0)=x_{2}(T)$, we know that there exists a point $t_{2} \in(0, T)$ such that $x_{2}^{\prime}\left(t_{2}\right)=0$. From the second equation of (3.1), Eqs. (3.8), (3.17), (3.18) and condition $\left(H_{1}\right)$, we obtain

$$
\begin{aligned}
\left\|x_{2}^{\prime}\right\| & \leq \frac{1}{2} \int_{0}^{T}\left|x_{2}^{\prime \prime}(t)\right| d t \\
& \leq \frac{\lambda}{2}\left(\int_{0}^{T}\left|f\left(t, x_{1}^{\prime}(t)\right)\right| d t+\int_{0}^{T}\left|g\left(t, x_{1}(t)\right)\right| d t+\int_{0}^{T}|e(t)| d t\right) \\
& \leq \lambda\left(N T+a T M_{1}^{p-1}+b T+T\|e\|\right):=\lambda M_{3} .
\end{aligned}
$$

Integrating the first equation of (3.1) over [0,T], we have $\int_{0}^{T} x_{2}(t) d t=\int_{0}^{T} \phi_{p}\left(x_{1}^{\prime \prime}(t)\right) d t=0$, which implies there is a point $t_{3} \in(0, T)$ such that $x_{2}\left(t_{3}\right)=0$, so

$$
\left\|x_{2}\right\| \leq \frac{1}{2} \int_{0}^{T}\left|x_{2}^{\prime}(t)\right| d t \leq \lambda T M_{3}:=\lambda M_{4}
$$

On the other hand, it follows from Eq. (3.2) and condition $\left(H_{4}\right)$ that

$$
\begin{aligned}
& \left(\phi_{p}\left(x_{1}^{\prime \prime}(t)\right)\right)^{\prime \prime}+\lambda^{p} f\left(t, x_{1}(t)\right) x_{1}^{\prime}(t)+\lambda^{p}\left(g_{0}\left(x_{1}(t-\delta(t))\right)+g_{1}\left(t, x_{1}(t-\delta(t))\right)\right. \\
& \quad=\lambda^{p} e(t) .
\end{aligned}
$$


Let $\xi \in[0, T]$ be as in Eq. (3.4), for any $t \in[\xi, T]$. Multiplying both sides of Eq. (3.21) by $x_{1}^{\prime}(t-\delta(t))\left(1-\delta^{\prime}(t)\right)$ and integrating on $[\xi, t]$ we get

$$
\begin{aligned}
\lambda^{p} & \int_{x_{1}(\xi-\delta(\xi))}^{x_{1}(t-\delta(t))} g_{0}(u) d u \\
= & \lambda^{p} \int_{\xi}^{t} g_{0}\left(x_{1}(s-\delta(s))\right) x_{1}^{\prime}(s-\delta(s))\left(1-\delta^{\prime}(s)\right) d s \\
= & -\int_{\xi}^{t}\left(\phi_{p}\left(x_{1}^{\prime \prime}(s)\right)\right)^{\prime \prime} x_{1}^{\prime}(s-\delta(s))\left(1-\delta^{\prime}(s)\right) d s \\
& -\lambda^{p} \int_{\xi}^{t} f\left(s, x_{1}^{\prime}(s)\right) x_{1}^{\prime}(s-\delta(s))\left(1-\delta^{\prime}(s)\right) d s \\
& -\lambda^{p} \int_{\xi}^{t} g_{1}\left(s, x_{1}(s-\delta(s))\right) x_{1}^{\prime}(s-\delta(s))\left(1-\delta^{\prime}(s)\right) d s \\
& +\lambda^{p} \int_{\xi}^{t} e(s) x_{1}^{\prime}(s-\delta(s))\left(1-\delta^{\prime}(s)\right) d s .
\end{aligned}
$$

Furthermore, by Eqs. (3.16), (3.17), (3.18) and (3.19), we have

$$
\begin{aligned}
\lambda^{p} \mid & \int_{x_{1}(\xi-\delta(\xi))}^{x_{1}(t-\delta(t))} g_{0}(u) d u \mid \\
= & \mid-\int_{\xi}^{t}\left(\phi_{p}\left(x_{1}^{\prime \prime}(s)\right)\right)^{\prime \prime} x_{1}^{\prime}(s-\delta(s))\left(1-\delta^{\prime}(s)\right) d s \\
& -\lambda^{p} \int_{\xi}^{t} f\left(s, x_{1}^{\prime}(s)\right) x_{1}^{\prime}(s-\delta(s))\left(1-\delta^{\prime}(s)\right) d s \\
& -\lambda^{p} \int_{\xi}^{t} g_{1}\left(s, x_{1}(s-\delta(s))\right) x_{1}^{\prime}(s-\delta(s))\left(1-\delta^{\prime}(s)\right) d s \\
& +\lambda^{p} \int_{\xi}^{t} e(s) x_{1}^{\prime}(s-\delta(s))\left(1-\delta^{\prime}(s)\right) d s \mid \\
\leq & \left.\left(1+\delta^{\prime}\right)\left\|x_{1}^{\prime}\right\| \int_{0}^{T} \mid \phi_{p}\left(x_{1}^{\prime \prime}(s)\right)\right)^{\prime \prime}\left|d s+\lambda^{p}\left(1+\delta^{\prime}\right)\left\|x_{1}^{\prime}\right\| \int_{0}^{T}\right| f\left(s, x_{1}^{\prime}(s)\right) \mid d s \\
& +\lambda^{p}\left(1+\delta^{\prime}\right)\left\|x_{1}^{\prime}\right\| \int_{0}^{T}\left|g_{1}\left(s, x_{1}(s-\delta(s))\right)\right| d s+\lambda^{p}\left(1+\delta^{\prime}\right)\left\|x_{1}^{\prime}\right\| \int_{0}^{T}|e(s)| d s \\
\leq & \left.\lambda^{p}\left(1+\delta^{\prime}\right) M_{2}\left(\int_{0}^{T} \mid f\left(s, x^{\prime}(s)\right)\right)\left|d s+\int_{0}^{T}\right| g\left(s, x_{1}(s)\right)\left|d s+\int_{0}^{T}\right| e(s) \mid d s\right) \\
& +\lambda^{p}\left(1+\delta^{\prime}\right)\left(M_{2} N T+M_{2}\left\|g_{M_{1}}\right\| T+M_{2}\|e\| T\right) \\
\leq & \lambda^{p}\left(1+\delta^{\prime}\right) M_{2}\left(M_{3}+N T+\left\|g_{M_{1}}\right\| T+\|e\| T\right),
\end{aligned}
$$

where $g_{M_{1}}=\max _{0 \leq x \leq M_{1}}\left|g_{1}(t, x)\right| \in L^{2}(0, T)$ are as in condition $\left(H_{4}\right)$. According to the singular condition $\left(H_{5}\right)$, we know that there exists a positive constant $M_{5}$ such that

$$
x_{1}(t-\delta(t)) \geq M_{5}, \quad \forall t \in[\xi, T]
$$

The case $t \in[0, \xi]$ (i.e., $\left.x_{1}(t-\delta(t)) \in[-\delta(0), \xi-\delta(\xi)]\right)$ can be treated similarly. 
From Eqs. (3.16), (3.17), (3.18), (3.19) and (3.24), we let

$$
\Omega=\left\{x=\left(x_{1}, x_{2}\right)^{\top}: E_{1} \leq x_{1}(t) \leq E_{2},\left\|x_{1}^{\prime}\right\| \leq E_{3},\left\|x_{2}\right\| \leq E_{4} \text { and }\left\|x_{2}^{\prime}\right\| \leq E_{5}, \forall t \in[0, T]\right\},
$$

where $0<E_{1}<\min \left(M_{5}, D_{1}\right), E_{2}>\max \left(M_{1}, D_{2}\right), E_{3}>M_{2}, E_{4}>M_{4}$ and $E_{5}>M_{3} . \Omega_{2}=\{x: x \in$ $\partial \Omega \cap \operatorname{Ker} L\}$ then $\forall x \in \partial \Omega \cap \operatorname{Ker} L$

$$
Q N x=\frac{1}{T} \int_{0}^{T}\left(\begin{array}{c}
\phi_{q}\left(x_{2}(t)\right) \\
-f\left(t, x_{1}^{\prime}\right)-g\left(t, x_{1}\right)+e(t)
\end{array}\right) d t .
$$

If $Q N x=0$, then $x_{2}(t)=0, x_{1}=E_{2}$ or $E_{1}$. But if $x_{1}(t)=E_{2}$, we know

$$
0=\int_{0}^{T}\left(g\left(t, E_{2}\right)-e(t)\right) d t
$$

From condition $\left(H_{2}\right)$, we have $x_{1}(t) \leq D_{2} \leq E_{2}$, which yields a contradiction. Similarly if $x_{1}=E_{1}$. We also have $Q N x \neq 0$, i.e., $\forall x \in \partial \Omega \cap \operatorname{Ker} L, x \notin \operatorname{Im} L$, so assumptions (1) and (2) of Lemma 2.1 are both satisfied. Define the isomorphism $J: \operatorname{Im} Q \rightarrow \operatorname{Ker} L$ as follows:

$$
J\left(x_{1}, x_{2}\right)^{\top}=\left(x_{2},-x_{1}\right)^{\top}
$$

Let $H(\mu, x)=-\mu x+(1-\mu) J Q N x,(\mu, x) \in[0,1] \times \Omega$, then $\forall(\mu, x) \in(0,1) \times(\partial \Omega \cap \operatorname{Ker} L)$,

$$
H(\mu, x)=\left(\begin{array}{c}
-\mu x_{1}-\frac{1-\mu}{T} \int_{0}^{T}\left(g\left(t, x_{1}\right)-e(t)\right) d t \\
-\mu x_{2}-(1-\mu) \phi_{q}\left(x_{2}\right)
\end{array}\right) .
$$

From condition $\left(H_{2}\right)$, we get $x^{\top} H(\mu, x) \neq 0, \forall(\mu, x) \in(0,1) \times(\partial \Omega \cap \operatorname{Ker} L)$. Hence

$$
\begin{aligned}
\operatorname{deg}\{J Q N, \Omega \cap \operatorname{Ker} L, 0\} & =\operatorname{deg}\{H(0, x), \Omega \cap \operatorname{Ker} L, 0\} \\
& =\operatorname{deg}\{H(1, x), \Omega \cap \operatorname{Ker} L, 0\} \\
& =\operatorname{deg}\{I, \Omega \cap \operatorname{Ker} L, 0\} \neq 0 .
\end{aligned}
$$

So assumption (3) of Lemma 2.1 is satisfied. By applying Lemma 2.1, we conclude that the equation $L x=N x$ has a solution $x=\left(x_{1}, x_{2}\right)^{\top}$ on $\bar{\Omega} \cap D(L)$, i.e., Eq. (2.1) has a $T$-periodic solution $x_{1}(t)$.

Next, we investigate the existence of a positive $T$-periodic solution for Eq. (1.1) with a singularity of attractive type.

Proof of Theorem 1.5 We follow the same strategy and notation as in the proof of Theorem 1.1. From Eq. (3.3) and condition $\left(H_{6}\right)$, we know that there are points $\tau, v \in(0, T)$ such that

$$
x_{1}(\tau-\delta(\tau)) \geq D_{3}, \quad x_{1}(v) \leq D_{4} .
$$


Next, we consider $\int_{0}^{T}\left|g\left(t, x_{1}(t-\delta(t))\right)\right| d t$. From Eq. (3.11) and conditions $\left(H_{1}\right),\left(H_{7}\right)$, we obtain

$$
\begin{aligned}
\int_{0}^{T}\left|g\left(t, x_{1}(t-\delta(t))\right)\right| d t= & \int_{g\left(t, x_{1}\right) \geq 0} g\left(t, x_{1}(t-\delta(t))\right) d t \\
& -\int_{g\left(t, x_{1}\right) \leq 0} g\left(t, x_{1}(t-\delta(t))\right) d t \\
= & -2 \int_{g\left(t, x_{1}\right) \leq 0} g\left(t, x_{1}(t-\delta(t))\right) d t \\
& -\int_{0}^{T} f\left(t, x_{1}^{\prime}(t)\right) d t+\int_{0}^{T} e(t) d t \\
\leq & 2 a^{\prime} \int_{0}^{T} x_{1}^{p-1}(t-\delta(t)) d t+2 b^{\prime} T \\
& +\int_{0}^{T}\left|f\left(t, x_{1}^{\prime}(t)\right)\right| d t+\int_{0}^{T}|e(t)| d t \\
= & 2 a^{\prime} \int_{0}^{T}\left|x_{1}(t-\delta(t))\right|^{p-1} d t+2 b^{\prime} T+N T+\|e\| T \\
\leq & \frac{2 a^{\prime}}{1-\delta^{\prime}} \int_{0}^{T}\left|x_{1}(t)\right|^{p-1} d t+2 b^{\prime} T+N T+\|e\| T .
\end{aligned}
$$

The proof further is the same as Theorem 1.1.

\section{Examples}

In this section, we present two examples to illustrate Theorems 1.1 and 1.5.

Example 4.1 Consider the fourth-order Rayleigh equation with singularity of repulsive type and time-dependent deviating argument:

$$
\begin{aligned}
& \left(\phi_{p}\left(x^{\prime \prime}(t)\right)\right)^{\prime \prime}+\left(\sin ^{2} t+5\right) \cos x^{\prime}(t)+\frac{1}{20 \pi}(\sin 2 t+3) x\left(t-\frac{1}{4} \sin 2 t\right)-\frac{1}{x^{\mu}\left(t-\frac{1}{4} \sin 2 t\right)} \\
& \quad=e^{\cos 2 t}
\end{aligned}
$$

where $\mu \geq 1$ and $p=4$.

It is clear that $T=\pi, f\left(t, x^{\prime}\right)=\left(\sin ^{2} t+5\right) \cos x^{\prime}(t), g(t, x)=\frac{1}{20 \pi}(\sin 2 t+3) x-\frac{1}{x^{\mu}}, \delta(t)=$ $\frac{1}{4} \sin 2 t, a=\frac{1}{5 \pi}, \delta^{\prime}=\frac{1}{2}<1, \pi_{4}=\frac{2 \pi(p-1)^{\frac{1}{p}}}{p \sin (\pi / p)}=\frac{2 \pi(4-1)^{\frac{1}{4}}}{4 \cdot \frac{\sqrt{2}}{2}}=\pi \times\left(\frac{3}{4}\right)^{\frac{1}{4}}$. It is obviously that $\left(H_{1}\right)-$ $\left(H_{5}\right)$ hold. Now we consider

$$
\frac{a T}{1-\delta^{\prime}}\left(\frac{T}{\pi_{p}}\right)^{2 p-1}=\frac{2}{5 \pi} \times \pi\left(\frac{\pi}{\pi \times\left(\frac{3}{4}\right)^{\frac{1}{4}}}\right)^{7}=\frac{2}{5} \times\left(\frac{4}{3}\right)^{\frac{7}{4}} \approx 0.6618<1 .
$$

Therefore, by Theorem 1.1, we know that Eq. (4.1) has at least one positive $\pi$-periodic solution. 
Example 4.2 Consider the fourth-order Rayleigh equation with singularity of attractive type:

$$
\begin{aligned}
& x^{(4)}(t)-\left(\cos ^{2}(2 t)+100\right) \sin x^{\prime}(t)-\frac{1}{3 \pi}(\sin 4 t+5) x\left(t-\frac{1}{8} \cos 4 t\right)+\frac{5}{x^{k^{\prime}}\left(t-\frac{1}{8} \cos 4 t\right)} \\
& =e^{\sin ^{2}(2 t)},
\end{aligned}
$$

where $\kappa^{\prime} \geq 1$ and $p=2$.

It is clear that $T=\frac{\pi}{2}, f\left(t, x^{\prime}\right)=-\left(\cos ^{2}(2 t)+100\right) \sin x^{\prime}, g(t, x)=-\frac{1}{3 \pi}(\sin 4 t+5) x+\frac{5}{x^{\kappa^{\prime}}}$, $\delta=\frac{1}{8} \cos 4 t, \delta^{\prime}=\frac{1}{2}, \pi_{2}=\frac{2 \pi(p-1)^{\frac{1}{p}}}{p \sin (\pi / p)}=\frac{2 \pi(2-1)^{\frac{1}{2}}}{4 \times \frac{1}{2}}=\pi$. Take $N=6, a^{\prime}=\frac{2}{\pi}, b^{\prime}=1$. It is obvious that $\left(H_{1}\right),\left(H_{4}\right),\left(H_{6}\right)-\left(H_{8}\right)$ hold. Now we consider

$$
\frac{a^{\prime} T}{1-\delta^{\prime}}\left(\frac{T}{\pi_{p}}\right)^{2 p-1}=\frac{4}{\pi} \times \frac{\pi}{2} \times\left(\frac{1}{2}\right)^{3}=\frac{1}{4}<1 .
$$

Therefore, applying Theorem 1.5, we know that Eq. (4.2) has at least one positive $\frac{\pi}{2}$ periodic solution.

\section{Conclusions}

In this paper, we introduce the existence of a positive $T$-periodic solution for the fourthorder $p$-Laplacian singular Rayleigh equation with time-dependent deviating argument. The nonlinear function has a time-dependent deviating argument. This implies that the work on estimating lower bounds of periodic solutions for Eq. (1.1) is more difficult than the corresponding work on Eq. (1.4) in [21]. Secondly, attractive conditions $\left(H_{6}\right),\left(H_{7}\right)$ and $\left(H_{8}\right)$ are in contradiction with the repulsive conditions $\left(H_{2}\right),\left(H_{3}\right)$ and $\left(H_{5}\right)$, and the methods of singularity of repulsive type are no longer applicable to the proof of a periodic solution for Eq. (1.1) with singularity of attractive type. In this paper, by using coincidence degree theory and conditions $\left(H_{1}\right)-\left(H_{5}\right)$, we prove the existence of a positive $T$-periodic solution for Eq. (1.1) with a singularity of repulsive type; applying conditions $\left(H_{1}\right),\left(H_{4}\right)$, $\left(H_{6}\right)-\left(H_{8}\right)$, we find that Eq. (1.1) with singularity of attractive type has at least one positive $T$-periodic solution.

Acknowledgements

YX and HML are grateful to anonymous referees for their constructive comments and suggestions, which have greatly improved this paper.

\section{Funding}

This work was supported by Education Department of Henan Province project (No. 16B110006) and Henan Polytechnic University Outstanding Youth Fund (J2016-03)

\section{Abbreviations}

Not applicable.

Availability of data and materials

Not applicable.

Ethics approval and consent to participate

YX and HML contributed to each part of this study equally and declare that they have no competing interests.

Competing interests

YX and HML declare that they have no competing interests. 
Authors' contributions

YX and HML contributed equally and significantly in writing this article. Both authors read and approved the final manuscript.

\section{Publisher's Note}

Springer Nature remains neutral with regard to jurisdictional claims in published maps and institutional affiliations.

Received: 20 June 2018 Accepted: 10 September 2018 Published online: 11 October 2018

\section{References}

1. Gaines, R., Mawhin, J.: Coincidence Degree, and Nonlinear Differential Equations. Lecture Notes in Mathematics, vol. 568. Springer, Berlin (1977)

2. Habets, P., Torres, P.: Some multiplicity results for periodic solutions of a Rayleigh differential equation. Dyn. Contin. Discrete Impuls. Syst. 8, 335-351 (2001)

3. Cheung, W., Ren, J.: Periodic solutions for $p$-Laplacian Rayleigh equations. Nonlinear Anal. 65, 2003-2012 (2006)

4. Cheng, Z., Ren, J.: Periodic solutions for a fourth-order Rayleigh type p-Laplacian delay equation. Nonlinear Anal. 70 516-523 (2009)

5. Ren, J., Yu, L., Siegmund, S.: Bifurcations and chaos in a discrete predator-prey model with Crowley-Martin functional response. Nonlinear Dyn. 90, 19-41 (2017)

6. Ma, T.: Periodic solutions of Rayleigh equations via time-maps. Nonlinear Anal. 75, 4137-4144 (2012)

7. Wang, Z:: On the existence of periodic solutions of Rayleigh equations. Z. Angew. Math. Phys. 56, 592-608 (2005)

8. Du, B., Lu, S.: On the existence of periodic solutions to a p-Laplacian Rayleigh equation. Indian J. Pure Appl. Math. 40, 253-266 (2009)

9. Sun, X., Yu, P., Qin, B.: Global existence and uniqueness of periodic waves in a population model with density-dependent migrations and Allee effect. Int. J. Bifurc. Chaos Appl. Sci. Eng. 27, 1-10 (2017)

10. Wang, L., Shao, J.: New results of periodic solutions for a kind of forced Rayleigh-type equations. Nonlinear Anal., Real World Appl. 11, 99-105 (2010)

11. Cheng, Z., Ren, J.: Periodic and subharmonic solutions for Duffing equation with singularity. Discrete Contin. Dyn. Syst., Ser. A 32, 1557-1574 (2012)

12. Chu, J., Torres, P., Zhang, M.: Periodic solution of second order non-autonomous singular dynamical systems. J. Differ. Equ. 239, 196-212 (2007)

13. Fonda, A., Manásevich, R., Zanolin, F.: Subharmonics solutions for some second order differential equations with singularities. SIAM J. Math. Anal. 24, 1294-1311 (1993)

14. Habets, P., Sanchez, L.: Periodic solutions of some Liénard equations with singularities. Proc. Am. Math. Soc. 109, 1135-1144 (1990)

15. Lu, S.: A new result on the existence of periodic solutions for Liénard equation with a singularity of repulsive type. J. Inequal. Appl. 2017, 37 (2017)

16. Ren, J., Cheng, Z., Chen, Y.: Existence results of periodic solutions for third-order nonlinear singular differential equation. Math. Nachr. 286, 1022-1042 (2013)

17. Sun, X., Zhao, L.: Perturbations of a class of hyper-elliptic Hamiltonian systems of degree seven with nilpotent singular points. Appl. Math. Comput. 289, 194-203 (2016)

18. Wang, H.: Positive periodic solutions of singular systems with a parameter. J. Differ. Equ. 249, 2986-3002 (2010)

19. Zhang, M.: Periodic solutions of Liénard equations with singular forces of repulsive type. J. Math. Anal. Appl. 203 , 254-269 (1996)

20. Wang, Z.: Periodic solutions of Liénard equation with a singularity and a deviating argument. Nonlinear Anal., Real World Appl. 16, 227-234 (2014)

21. Chen, L., Lu, S.: A new result on the existence of periodic solutions for Rayleigh equations with a singularity of repulsive type. Adv. Differ. Equ. 2017, 106 (2017)

22. Cheng, Z., Bi, Z., Yao, S.: Periodic solution for p-Laplacian Rayleigh equation with attractive singularity and time-dependent deviating argument. Bound. Value Probl. 2018, 20 (2018)

23. Guo, Y., Wang, Y., Zhou, D.: A new result on the existence of periodic solutions for Rayleigh equation with a singularity. Adv. Differ. Equ. 2017, 394 (2017)

24. Wang, Z., Ma, T.: Periodic solutions of Rayleigh equations with singularities. Bound. Value Probl. 2015, 154 (2015)

25. Zhang, M.: Nonuniform nonresonance at the first eigenvalue of the $p$-Laplacian. Nonlinear Anal. TMA 29, 41-51 (1997)

26. Torres, P., Cheng, Z., Ren, J.: Non-degeneracy and uniqueness of periodic solutions for $2 n$-order differential equation. Discrete Contin. Dyn. Syst., Ser. A 33, 2155-2168 (2013) 